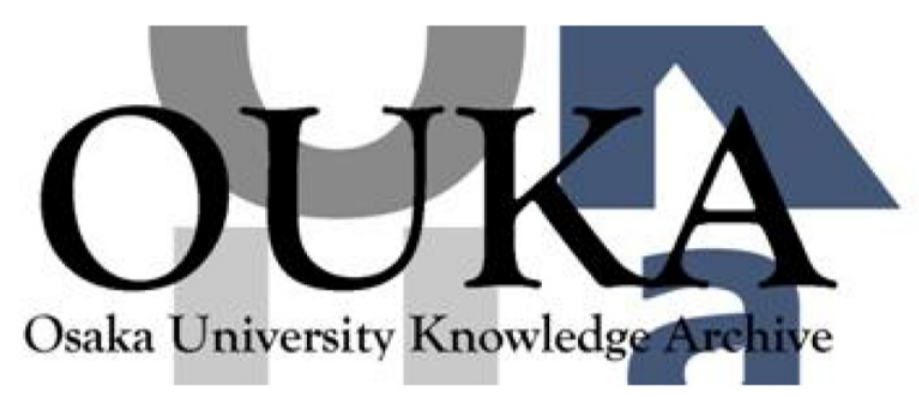

\begin{tabular}{|c|l|}
\hline Title & $\begin{array}{l}\text { Gelatin microspheres crosslinked with } \gamma \text {-ray : } \\
\text { Preparation, sorption of proteins, and } \\
\text { biodegradability }\end{array}$ \\
\hline Author(s) & $\begin{array}{l}\text { Terao, Ken; Karino, Tomotake; Nagasawa, } \\
\text { Naotsugu et al. }\end{array}$ \\
\hline Citation & $\begin{array}{l}\text { Journal of Applied Polymer Science. 91(5) } \\
\text { p. 3083-p.3087 }\end{array}$ \\
\hline Issue Date & $2004-03-05$ \\
\hline oaire:version & AM \\
\hline URL & https://hdl. handle.net/11094/81796 \\
\hline rights & \\
\hline Note & \\
\hline
\end{tabular}

Osaka University Knowledge Archive : OUKA

https://ir. Library. osaka-u. ac. jp/

Osaka University 


\title{
Gelatin microspheres crosslinked with gamma ray: preparation, sorption of proteins, and biodegradability
}

\author{
Ken Terao $^{1, *}$, Tomotake Karino ${ }^{1}$, Naotsugu Nagasawa ${ }^{2}$, Fumio Yoshii ${ }^{2}$, \\ Miyoko Kubo ${ }^{3}$, Toshiaki Dobashi ${ }^{1}$ \\ ${ }^{1}$ Department of Biological and Chemical Engineering, Faculty of Engineering, Gunma \\ University, Kiryu, Gunma 376-8515, Japan \\ ${ }^{2}$ Takasaki Radiation Research Establishment, Japan Atomic Energy Research Institute, \\ Watanuki-machi, Takasaki, Gunma 370-1292, Japan. \\ ${ }^{3}$ Department of Plastic and Reconstructive Surgery, Kawasaki Medical School, 577, \\ Matsushima, Kurashiki, Okayama 701-0192, Japan.
}

\section{Running head: BIODEGRADABILITY OF GELATIN MICROSPHERES}

Corresponding to K. Terao (terao@ @ce.gunma-u.ac.jp)

* Present address: Department of Chemistry, University of Tennessee, 552 Buehler Hall, Knoxville, Tennessee 37996.

\begin{abstract}
A new type of gelatin microspheres was manufactured with the crosslinking method using gamma-ray irradiation. Microspheres thus obtained were characterized by microscopic observation and enzymatic degradability. The mean diameter of the microspheres was in the range from 2.4 to $3.6 \mu \mathrm{m}$ and the size distribution was quite small. The size decreased with increasing the shear rate of the emulsifier used in the preparation and was not affected by the radiation dose. The enzymatic degradability decreased with increasing the radiation dose and decreasing the gelatin concentration of the microsphere. In other words, the rate of the enzymatic decomposition of microspheres can be controlled by these two parameters. These trends were consistent with our previous results for the gel sheet made of gelatin. Their sorption of proteins was also investigated with the use of three types of proteins labeled by fluorescent pigment. From the fluorescence micrographs it was observed that the gelatin microspheres adsorbed only proteins having the opposite charge.
\end{abstract}

Key words: gels; irradiation; crosslinking; biodegradable; proteins

\section{INTRODUCTION}

Microspheres prepared by crosslinking of polyelectrolytes are a group of useful carriers of the drug delivery system because they can be injected to the body and it is expected to adsorb many kinds of proteins by the electrostatic force of attraction. Indeed, Tabata et al. prepared microspheres consisting of gelatin crosslinked by glutaraldehyde and demonstrated the applicability as a carrier of the drug delivery system [1]. The crosslinking reagent, glutaraldehyde has, however, unneglectable toxicity and oncogenicity $[2,3]$.

Recently, we found that concentrated gelatin gels (physical gel) can be crosslinked by gamma-ray or electron beam [4]. We prepared gel sheets (chemical gel) by the irradiation of electron beam, and concluded that the enzymatic degradability of the sheet could be controlled over a wide range of degradation time by varying the gelatin concentration and the radiation dose. The hydrogel thus obtained is useful for some clinical medicine and/or food processing because no crosslinking reagents and organic solvents are required in the preparation process and it is expected that a release of polyelectrolytes from the gel could be controlled by the biodegradability of the hydrogel $[5,6]$. 
In this work, we manufactured hydrogel microspheres (physical gel) made of two kinds of gelatin samples originated from porcine skin (type A) and bovine skin (type B) and irradiated gamma ray to them in order to crosslink the gelatin molecules. Microscopic observation was carried out for the microspheres in order to determine the average size and the size distribution. Enzymatic degradability was estimated from the time course of optical absorbance from the suspension of the microspheres containing proteinase. The sorption of polypeptides was also investigated with acidic or basic proteins labeled with fluorescein-4-isothiocyanate. Selective sorption obtained from the experiment could be one of the most useful function of these microspheres.

\section{EXPERIMENTAL}

Materials, preparation of microspheres, and irradiation

Commercially obtained gelatin samples from porcine skin (type A) were purchased from Sigma Co. and used for this study without further purification. $3 \mathrm{~g}$ of gelatin solution (5 or $10 \mathrm{wt} \%$ in pure water) was mixed with cyclohexane containing a small amount of SY-glyster CR-310 (tetraglycerine polyrisinolate), a gift from Sakamoto Yakuhin Kogyo Co., as a surfactant. This surfactant has been approved as a non-toxic food additive. Each mixture was homogenized at 6,000 or $16,000 \mathrm{rpm}$ at $50^{\circ} \mathrm{C}$ for $15 \mathrm{~min}$ by an emulsifier (Excel Auto, Nihon Seiki Co.). The resultant suspension was cooled to room temperature and stored at low temperature (about $5^{\circ} \mathrm{C}$ ) until irradiation of gamma ray. The suspensions were irradiated with gamma ray generated from a ${ }^{60} \mathrm{Co}$ source (Takasaki Radiation Research Establishment) at a dose rate of $10 \mathrm{kGy} / \mathrm{h}$. Then the suspensions were freezed with liquid nitrogen and the gel spheres were separated with the conventional freeze-dry method.

In order to compare the biodegradability of the newly prepared gelatin microspheres with those crosslinked by other methods, we prepared two other microspheres as follows. Unirradiated microspheres prepared with the same method as described above were freeze-dried and irradiated by ultraviolet light in air at a distance of $20 \mathrm{~cm}$ from a UV lamp $(254 \mathrm{~nm}, 4 \mathrm{~W})$ for three days or soaked into $25 \%$ glutaraldehyde aqueous solution. The latter microspheres were separated by washing with ethanol several times and dried in vacuum.

\section{Size distribution of microspheres}

Size distribution measurements were carried out for gelatin microspheres dispersed in water using a Nikon Microphot-FXA microscope combined by $\times 10$ objective lens (Nikon). Micrographs were taken with a Nikon CCD camera unit. The diameter $d$ and the distribution of the microspheres were estimated from the micrographs and about 1,000 spheres (20 30 micrographs) were counted for each run.

\section{Enzymatic degradation}

Each enzymatic degradability measurement was carried out for the gelatin microspheres in $0.02 \mathrm{M}$ sodium bicarbonate / $0.001 \mathrm{M}$ calcium chloride buffer solutions with proteinase (Tokyo Kasei, from bacillus subtilis) of $0.01 \mathrm{~g} \mathrm{~cm}^{-3}$ as an enzyme at $40^{\circ} \mathrm{C}$. The activity of the enzyme was stated to be 89,000 units $\mathrm{g}^{-1}$ with using casein as a substrate according to the data sheet issued by the corporation. A small amount of microsphere dispersing liquid was poured into a $1 \mathrm{~cm}$ quartz cell filled with the proteinase solution. UV absorbance at $275 \mathrm{~nm}$ wavelength resulting from the aromatic amino acids consisting of the dissolved gelatin molecule was measured at appropriate time intervals. We note that the solution was stirred with a magnetic stirrer while conducting the measurement.

\section{Sorption of proteins to microspheres}

In order to examine if the microspheres adsorb proteins, microscopic fluorescence observations were performed under a Nikon Microphot-FXA microscope for the microspheres mixed proteins labeled with fluorescein-4-isothiocyanate (FITC-I, Dojin 
Laboratories). We chose an acidic protein, bovine serum albumin (BSA) purchased from Sigma Co. and two basic proteins, protamine sulfate purchased from Wako Co. and polylysine (Lot No. 2000306SF) gifted from Chisso Co. These proteins were reacted with FITC-I in the following manner: An aliquot of FITC-I aqueous solution was poured into protamine sulfate, or BSA, or polylysine aqueous solution and the mixture was stirred at $20^{\circ} \mathrm{C}$ for 24 hours. The isothiocyanate group of FITC-I was bonded to the amino groups of each protein in this process. In order to test the dependence of the type of gelatin, acidic or basic, the fluorescence observation was also carried out for the microspheres made of bovine skin gelatin (type B) purchased from Sigma Co.

\section{RESULTS AND DISCUSSION}

\section{Size distribution of microspheres}

Figure 1 shows the size distribution of the microspheres made of $5 \mathrm{wt} \%$ porcine gelatin solution. The size distribution curve is almost independent of the radiation dose, in contrast to considerable effects of the radiation dose on the gel fraction and the swelling shown in our previous study [4]. This apparent inconsistency results from the difference in the range of radiation dose; the radiation dose used in this work is quite large and the dose dependence of swelling behavior is negligibly small in this range. The size distribution of the microspheres made of $10 \mathrm{wt} \%$ gelatin solution is essentially the same as that of $5 \mathrm{wt} \%$ solution. Each size distribution of microspheres obeys the logalithmic normal distribution curve, and the mean value is determined to be $2.4 \mu \mathrm{m}$ and $3.6 \mu \mathrm{m}$ for those prepared by the emulsification at $16,000 \mathrm{rpm}$ and 6,000 rpm, respectively. The standard deviation is estimated to be 0.33 and 0.38 for $16,000 \mathrm{rpm}$ and $6,000 \mathrm{rpm}$, respectively. Thus, the mean size increases appreciably with decreasing emulsification rate. This trend is natural in the process of formation of emulsion. We note that the mean size of microspheres in water is not as large as that in cyclohexane, the original size of the emulsion, but the difference is quite small.

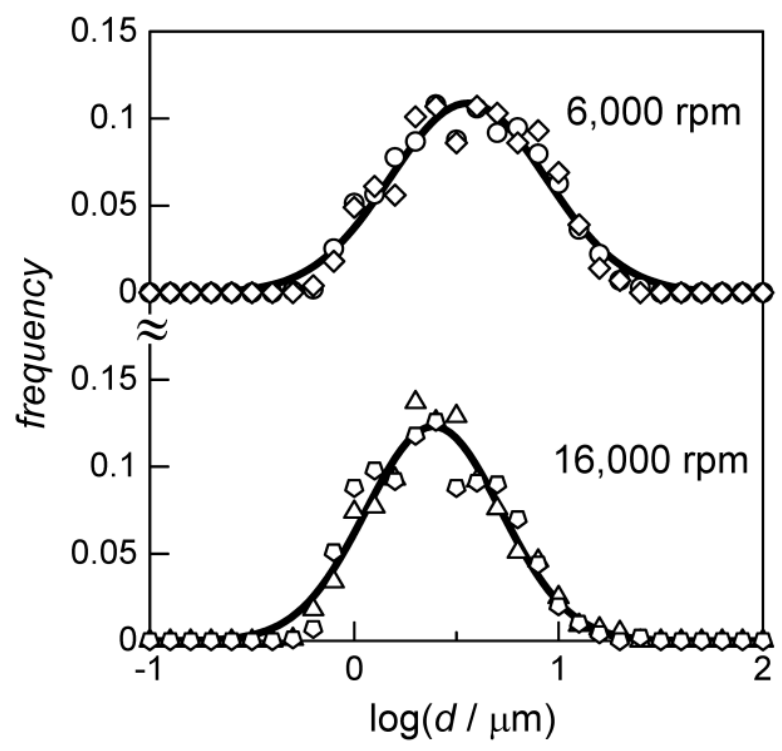

Figure 1. Droplet size distributions of the porcine gelatin microspheres in water. Circles: microspheres irradiated with $30 \mathrm{kGy}$ and homogenized at 6,000 rpm, squares: $60 \mathrm{kGy}$ and 6,000 rpm, triangles: $30 \mathrm{kGy}$ and 16,000 rpm, pentagon: $60 \mathrm{kGy}$ and 16,000 rpm. Curves were normal logalithmic curve with the mean value and the deviation listed in the text.

\section{Enzymatic biodegradation behavior}

Figure 2 illustrates the time course of the optical absorbance from the mixture of the proteinase and the microspheres made of gelatin from porcine skin. Data points near $t=0$ is 
complicated, but the entire curve is S-shaped and approaches each asymptotic value when $t$ is large. It is expected from these data that two effects, turbidity from the microspheres and absorbance from degraded gelatin molecules affect the total absorbance. In order to explain this complicated behavior of absorbance, we examined these effects by evaluating the absorbance $A$ of the solution under degradation (see Appendix A). The resultant expression for $A$ is expressed as the sum of absorbance $A_{\mathrm{g}}$ from degraded gelatin molecules and turbidity $\tau_{\mathrm{m}}$ of the microspheres, i. e.,

$$
\begin{array}{rlrl}
A & =A_{\mathrm{g}}+\tau_{\mathrm{m}} & t \leq \frac{r_{0}}{v} \\
& =A_{\infty} & t>\frac{r_{0}}{v}
\end{array}
$$

where

$$
\begin{aligned}
& A_{\mathrm{g}}=A_{\infty}\left[3\left(\frac{v t}{r_{0}}\right)-3\left(\frac{v t}{r_{0}}\right)^{2}+\left(\frac{v t}{r_{0}}\right)^{3}\right] \\
& \tau_{\mathrm{m}}=6 \pi K^{\prime} \int_{0}^{\pi} \frac{\left[\sin k\left(r_{0}-v t\right)-k\left(r_{0}-v t\right) \cos k\left(r_{0}-v t\right)\right]^{2}}{k^{6}}\left(1+\cos ^{2} \theta\right) \sin \theta \mathrm{d} \theta
\end{aligned}
$$

Here $r_{0}$ is the initial radius of the microsphere, $v$ is the rate of reducing radius, $K^{\prime}$ is the constant shown in eq A6, $A_{\infty}$ is the absorbance at infinitely long time, and $k$ is the absolute value of the scattering vector defined as

$k=\frac{4 \pi n_{0}}{\lambda_{0}} \sin \frac{\theta}{2}$

where $n_{0}$ and $\lambda_{0}$ are the refractive index of the solvent and the wavelength of the incident light in vacuum, respectively.

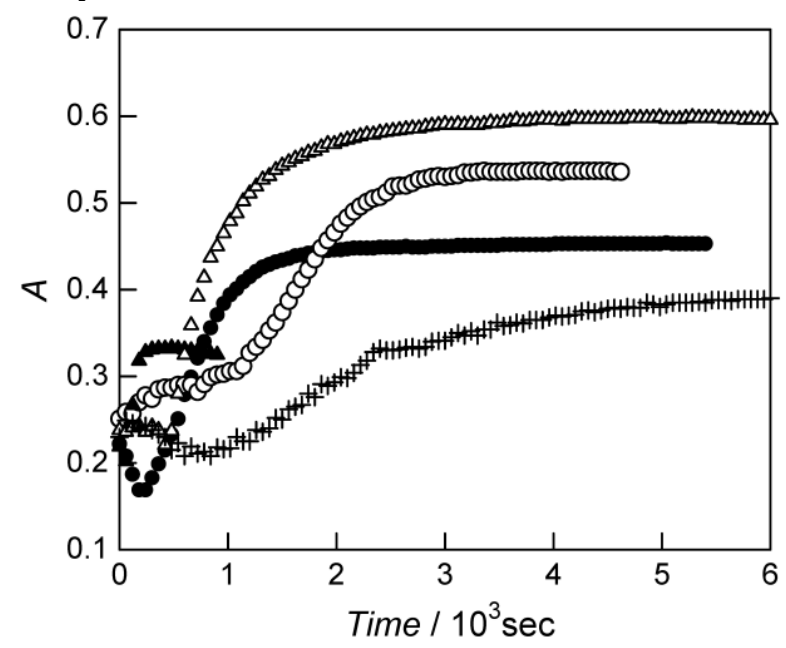

Figure 2. Time courses of the absorbance $A$ at $275 \mathrm{~nm}$ of the buffer solution including proteinase and gelatin microspheres irradiated with gamma-ray. Filled circles: microspheres made of $5 \mathrm{wt} \%$ solution, irradiated with $30 \mathrm{kGy}$, and homogenized with 16,000 rpm; unfilled circles: $5 \mathrm{wt} \%, 60 \mathrm{kGy}, 16,000 \mathrm{rpm}$; filled triangles: $10 \mathrm{wt} \%, 30 \mathrm{kGy}, 16,000 \mathrm{rpm}$; unfilled triangles: $10 \mathrm{wt} \%, 60 \mathrm{kGy}, 16,000 \mathrm{rpm}$; crosses: $5 \mathrm{wt} \%, 60 \mathrm{kGy}, 6,000 \mathrm{rpm}$. 
An example of theoretical calculations of eq 1 for $A_{\infty}=1, K^{\prime}=0.35 \mathrm{~g}^{-1} \mathrm{~cm}^{4}, n_{0}=1.33$, and $\lambda_{0}=0.273 \mu \mathrm{m}$ are illustrated in Figure 3. Panel (a) shows $A$ plotted against time $t$ for the same $v$ and a little different $r_{0}$. These theoretical calculations reproduce the complicated curve illustrated in Figure 2, at least qualitatively. However, a small difference in $r_{0}$ results in considerable change of $A$ in the small $t$ range. It is expected from this result that the polydispersity of microspheres affects serious transformation of the time course in this range. On the other hand, the time courses become independent of $r_{0}$ at large $t$. Panel (b) illustrates the plot of $A$ vs $t$ for the same $r_{0}$ and different $v$. It is seen that the difference of $v$ can be distinguished significantly from the time when $A$ approaches each asymptotic value. In other words, the difference of $v$ can be estimated from such data. In the light of this conclusion, the degradation rate increases with decreasing dose and increases with increasing concentration of gelatin. The same trend is observed for the microspheres made at low shear rate of $6,000 \mathrm{rpm}$. These results are consistent with our enzymatic degradation data [4] for gelatin sheets irradiated by electron beam. On the other hand, the degradation rate decreases with decreasing the rotation speed of the emulsifier at the preparation of microsphere (not shown). This is because the mean diameter of the microsphere increases with decreasing the rotation speed.

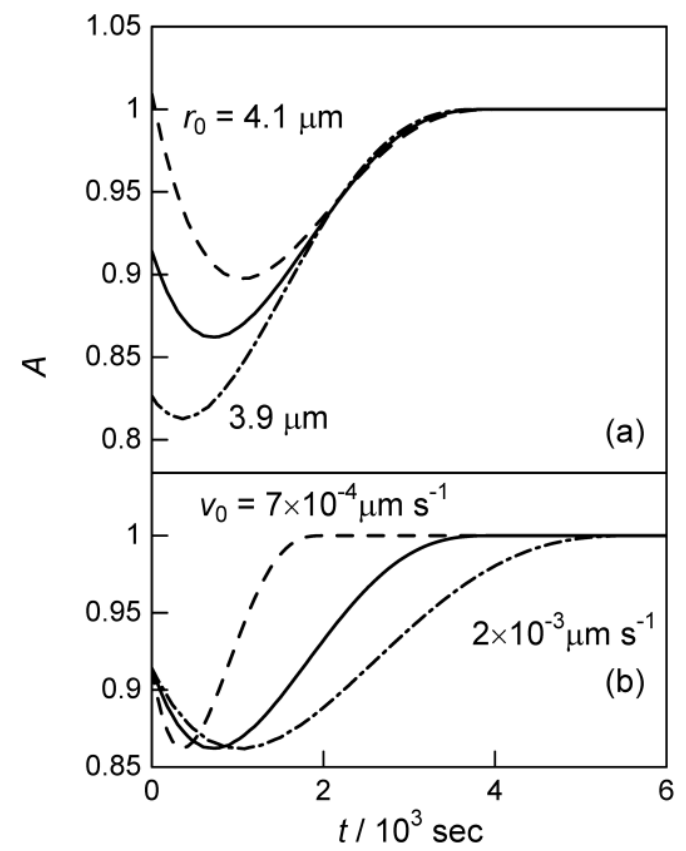

Figure 3. Theoretical time courses of absorbance $A$. Curves are calculated from eqs 1 and 2 with $A_{\infty}=1, K^{\prime}=0.35 \mathrm{~g}^{-1} \mathrm{~cm}^{4}, n_{0}=1.33$, and $\lambda_{0}=0.275 \mu \mathrm{m}$. Other parameters are indicated in the figure: (A) radius dependence, (B) degradation velocity dependence.

Figure 4 illustrates the comparison of the time courses thus obtained with those for the other microspheres crosslinked with glutaraldehyde or ultraviolet light. Compared with Figure 3, it is shown that the enzymatic degradability of the microspheres crosslinked with gamma ray is as changeable (or controllable) as those crosslinked with glutaraldehyde, and the degradability of those irradiated with ultraviolet light is much smaller. 


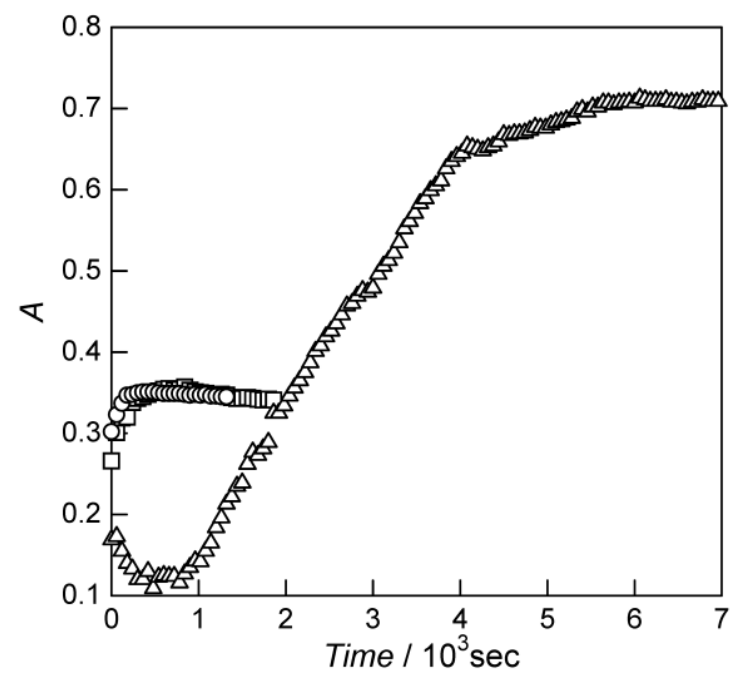

Figure 4. Time courses of the absorbance $A$ at $275 \mathrm{~nm}$ of the buffer solution including proteinase and gelatin microspheres. Circles: microspheres crosslinked with UV for three days; triangles: crosslinked with glutaraldehyde for 15 hours, squares: crosslinked with glutaraldehyde for $20 \mathrm{~min}$.

\section{Sorption of proteins to microspheres}

An example of a pair of micrograph is shown in Figure 5. These pictures were taken for the mixture of protamine sulfate labeled with FITC-I and the microspheres made of bovine skin gelatin. Panel (A) is a (normal) micrograph and many microspheres are observed. A lot of luminous points in Panel (B), fluorescent picture, are observed at the same positions of the microspheres in Panel (A). This observation indicates that protamine adhered to the microspheres in this case. In fact, the fluorescence did not disappear even after colored microspheres were washed in buffer solution several times. We judged if each protein adhered to microspheres from such pictures and summarized the results in Table 1. Here, BSA and type B gelatin (from bovine skin) are acidic proteins and have minus charges. On the contrary, protamine sulfate, polylysine, and type A gelatin (from porcine skin) are basic proteins and have plus charges. Therefore, it is concluded from this table that microspheres made of each type of gelatin adsorbed only proteins having opposite charge. Thus, the newly prepared gelatin microsphere has the same sorption properties as microspheres crosslinked by glutaraldehyde.

In conclusion, gelatin microspheres crosslinked by gamma ray without any chemical reagent have been manufactured. The average size microspheres can be controlled by emulsification speed in the preparation. Enzymatic degradability and polypeptide sorption properties are controllable as much as microspheres crosslinked by glutaraldehyde.

Table 1

Adsorptive Behavior of Proteins to Gelatin Microspheres

\begin{tabular}{lll}
\hline \multirow{2}{*}{ Protein } & \multicolumn{2}{l}{ Origin of gelatin (type) } \\
\cline { 2 - 3 } & Porcine skin (A) & Bovine skin (B) \\
\hline BSA & $\mathrm{O}$ & $\mathrm{X}$ \\
Protamine & $\mathrm{X}$ & $\mathrm{O}$ \\
Polylysine & $\mathrm{X}$ & $\mathrm{O}$ \\
\hline
\end{tabular}

O: Adsorptive, X: Not Adsorptive 

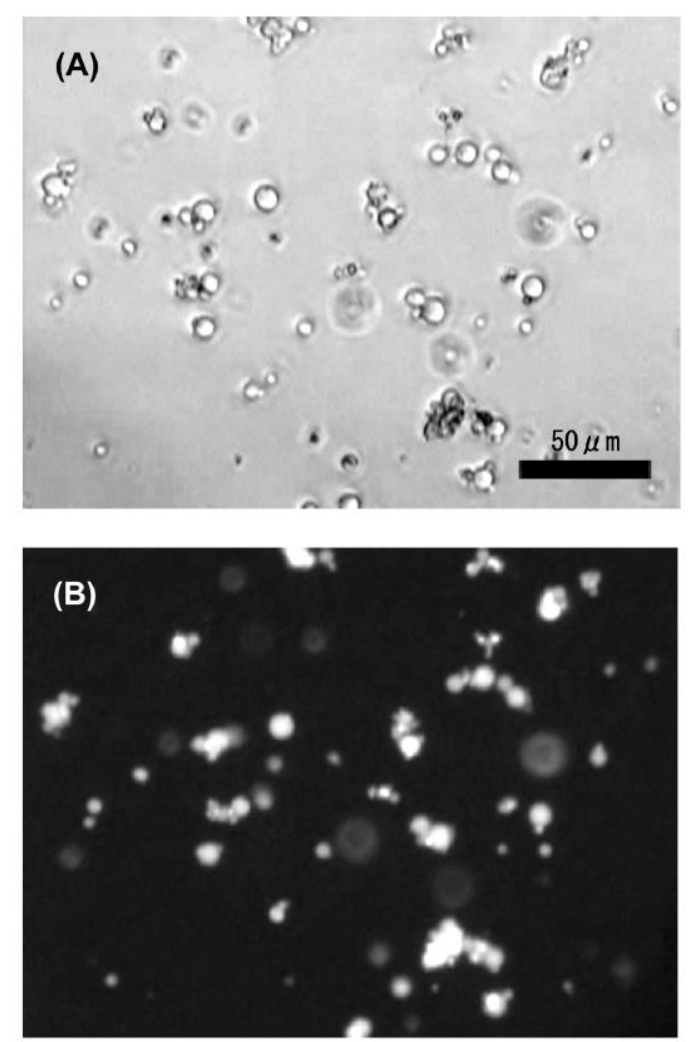

Figure 5. An example of the optical photomicrograph of the mixture of microspheres and the protein labeled with fluorescence in water. The microspheres in these pictures were made of $5 \mathrm{wt} \%$ solution of bovine gelatin, irradiated with $30 \mathrm{kGy}$, and homogenized at $16,000 \mathrm{rpm}$. (A) normal micrograph, (B) fluorescent micrograph.

\section{Appendix A.}

\section{Derivation of $A_{\mathrm{g}}$}

When the degradation of the gel microsphere occurs on the surface, the absorbance increment $d A_{\mathrm{g}}$ from a sphere dissolved in buffer is proportional to the surface area $S$.

$$
d A_{\mathrm{g}}=-\gamma l_{\mathrm{c}} V_{\mathrm{c}}^{-1} n C_{\mathrm{g}} S(r) d r=-4 \pi \lambda_{\mathrm{c}} V_{\mathrm{c}}^{-1} n C_{\mathrm{g}} r^{2} d r \quad 0 \leq r \leq r_{0}
$$

where $r$ is the radius of the sphere at $t, r_{0}$ is the original radius (the radius at $t=0$ ), $l_{\mathrm{c}}$ is the light-path length of the cell, $V_{\mathrm{c}}$ is the volume of the suspension in the cell, $n$ is the number of spheres in the cell, $C_{\mathrm{g}}$ is the gelatin concentration of the sphere, and $\gamma$ is a constant. The differential equation can be solved using a boundary condition $A_{\mathrm{g}}\left(r_{0}\right)=0$ as

$$
A_{\mathrm{g}}=\frac{4}{3} \pi \gamma l_{\mathrm{c}} V_{\mathrm{c}}^{-1} n C_{\mathrm{g}}\left(r_{0}^{3}-r^{3}\right) \quad 0 \leq r \leq r_{0}
$$

When $A_{\infty}$ is defined as $A_{\mathrm{g}}$ at $r=0$. If the gel is degraded with a uniform velocity $v, r(t)$ is expressed as

$$
\begin{aligned}
r(t) & =r_{0}-v t & & 0 \leq t \leq r_{0} / v \\
& =0 & & t>r_{0} / v
\end{aligned}
$$

Substitution of eq A3 into eq A2 leads eq 2. 


\section{Derivation of $\tau_{\mathrm{m}}$}

The turbidity $\tau_{\mathrm{m}}$ from the microspheres can be expressed as the total sum of the intensity of the scattered light as

$$
\tau_{\mathrm{m}}=2 \pi \int_{0}^{\pi} R_{\theta}\left(1+\cos ^{2} \theta\right) \sin \theta \mathrm{d} \theta
$$

where $\theta$ and $R_{\theta}$ represent the scattering angle and the Rayleigh ratio of the suspension, respectively. When the light scattering intensity can be assumed to represent with the particle scattering function $P(\theta)$ for rigid spheres, $R_{\theta}$ is expressed as

$$
\begin{aligned}
& R_{\theta}=K c M P(\theta)=K^{\prime} r^{6} P(\theta)=\frac{3 K^{\prime}(\sin k r-k r \cos k r)^{2}}{k^{6}} \\
& K^{\prime}=16 \pi^{2} K n C_{\mathrm{g}}^{2} N_{\mathrm{A}} / 9 V_{\mathrm{c}}
\end{aligned}
$$

where $K$ is the optical constant, $c$ is the mass concentration of gelatin in the suspension and equal to $4 \pi r^{3} n C_{\mathrm{g}} / 3 V_{\mathrm{c}}, M$ is the molecular weight of a microsphere without water, and $N_{\mathrm{A}}$ is the Avogadro number. Substitution of eqs A3 and A5 into eq A4 leads eq 3.

\section{References}

1. Y. Tabata, and Y. Ikada, Adv. Drug Delivery Rev. 31, 287 (1998).

2. B. Ballantyne, S. L. Jordan, J. Appl. Toxicol. 21, 131 (2001).

3. J. P. Van Miller, S. J. Hermansky, P. E. Losco, B. Ballantyne, Toxicol. 175, 177 (2002).

4. K. Terao, N. Nagasawa, H. Nishida, K. Furusawa, Y. Mori, T. Dobashi, and F. Yoshii, J. Biomater. Sci. Polym. Edn, in press.

5. Y. Tabata, A. Nagano, M. Muniruzzaman, and Y. Ikada, Biomaterials, 19, 1781 (1998).

6. M. Yamamoto, Y. Ikada, Y. Tabata, and J. Biomater. Sci. Polymer Edn., 12, 77 (2001). 